\title{
Review of the Theory of Pulsar Wind Nebulae
}

\author{
N. Bucciantini ${ }^{1,2, \star}$ \\ 1 INAF, Osservatorio Astrofisico di Arcetri, L.go E. Fermi 5, 50125, Firenze, Italy \\ 2 INFN, Sezione di Firenze, via G. Sansone 1, 50019, Sesto Fiorentino (FI), Italy
}

Received 30 July 2013, accepted 25 September 2013

Published online later

Key words pulsars: general - magnetohydrodynamics - radiation mechanism: non-thermal - stars: winds, outflows supernova remnants

\begin{abstract}
Pulsar Wind Nebulae (PWNe) are ideal astrophysical laboratories where high energy relativistic phenomena can be investigated. They are close, well resolved in our observations, and the knowledge derived in their study has a strong impact in many other fields, from AGNs to GRBs. Yet there are still unresolved issues, that prevent us from a full clear understanding of these objects. The lucky combination of high resolution X-ray imaging and numerical codes to handle the outflow and dynamical properties of relativistic MHD, has opened a new avenue of investigation that has lead to interesting progressed in the last years. Despite all of these, we do not understand yet how particles are accelerated, and the functioning of the pulsar wind and pulsar magnetosphere, that power PWNe. I will review what is now commonly known as the MHD paradigm, and in particular I will focus on various approaches that have been and are currently used to model these systems. For each I will highlight its advantages and limitations, and degree of applicability.
\end{abstract}

(C) 2013 WILEY-VCH Verlag GmbH \& Co. KGaA, Weinheim

\section{Introduction}

Pulsar Wind Nebulae (PWNe) form when the ultrarelativistic wind from a pulsar interacts with the ambient medium, either the parent Supernova Remnant (SNR) or the ISM (Bucciantini 2008; Chevalier 2004; Gaensler and Slane 2006). They are bubbles of relativistic particles, pairs and perhaps ions, emitting in a broad band spectrum from radio to $\gamma$-rays, via synchrotron and Inverse Compton emission (Asaoka and Koyama 1990; Bandiera 2000; Harding 1996; Kargaltsev and Pavlov 2010; Weiler and Panagia 1978). The best example of a PWN, often considered the prototype of this entire class of objects, is the Crab Nebula (Hester 2008). It is such a well known system, that the majority of our models are tuned to address its properties.

There are two key ingredients that go into any good recipe for cooking a realistic model of a PWN. The first ingredient is the acceleration mechanism responsible for producing the non-thermal particle's distribution function that is observed. The second ingredient is the dynamical model of the PWN, describing the advection and evolution of the flow within these systems.

The problem of accelerating the observed particle's distribution function is a long standing one (Arons and Tavani 1994; Bykov and Treumann 2011). Recent particle-incell simulations, in a variety of different conditions, have not helped to figure out a satisfactory solution. Ions have been invoked, but they must carry a substantial fraction of the total spin-down energy, for which there is no clear

\footnotetext{
^ Corresponding author: e-mail: niccolo@ arcetri.astro.it
}

evidence (Amato and Arons 2006; Sironi and Spitkovsky 2011b). Efficient Fermi-like acceleration of a pure pair plasma requires very small magnetizations in the upstream pulsar wind, that are reached only in a small solid angle (Sironi and Spitkovsky 2009). Reconnection of a striped wind (Lyubarsky and Liverts 2008; Lyubarsky 2003; Sironi and Spitkovsky 2011a), can provide hard spectra, compatible with the radio emission, but requires very high multiplicity and small Lorentz factors, contrasting the expectations for pulsar wind dynamics (Bogovalov 1997; Goldreich and Julian 1969; Hibschman and Arons 2001). There is also the possibility of continuous distributed turbulent acceleration in the bulk of the nebula (Barnes and Scargle 1973; Bucciantini et al. 2011). There is however a general consensus, based on the wavelength dependent size of these objects, that X-ray emitting particles must be accelerated at the wind termination shock. The origin of radio emitting ones is still currently debated (Atoyan and Aharonian 1996; Olmi et al. Private communication).

On the other hand dynamical models for the flow properties have developed to a greater success. There is now a well established paradigm, based on MHD. The key assumption is that the Larmor radius of the vast majority of the particles is much smaller that the typical size of these nebulae. This is true up to the highest observed energies. This allows one to use a fluid description, that can easily be formulated in terms of MHD. Once coupled with a prescription for particle's acceleration, it can easily be turned into an emission model. In principle, a satisfactory model, built along these lines, should be able to relate the observed 
morphology to the pulsar wind properties, and to provide a unifying picture for all these objects.

Recently, the tools and approches that have been used in the investigation of PWNe have been applied to Long and Short GRBs (Bucciantini et al. 2012, 2008, 2009; Dall'Osso et al. 2011). Indeed these systems too can be modeled as expanding relativistic magnetized bubbles, and share a large amount of physics and dynamics with PWNe.

I will review in the following the various approaches that have been presented in the literature to model these systems. I will start from the simpler 1-Zone and 1-D models and show how the extension to higher dimensions can both provide a more accurate description and also raise new questions. I will conclude with a brief review of various models for pulsar bow-shock nebulae.

\section{1-Zone Models}

With the label 1-Zone model (or equivalently $0+1$ model), we refer to any model where the PWN is described as an object, whose geometry is only characterized by a typical size (often its radius), which in principle can also depend on time. These models completely neglect the possibility of internal structure in the PWN and assume that all quantities are uniform. Despite the simplicity of this approach, these models have proved very successful for the investigation of the global rough properties of these systems. In particular they have been applied to the study of the evolution of PWNe, often in conjunction with their interaction with the partent SNR. The first 1-Zone model for PWNe was put forward by Pacini and Salvati (1973). Here the evolution of a non-thermal distribution of particles, inside an expanding bubble, subject to adiabatic losses, radiation losses and a continuous injection, was investigated, in relation to the non-thermal emission of the Crab Nebula. All the key ideas of 1-Zone models were already laid down. The analytical approach of this seminal work however, required assumptions on the temporal evolution of the various quantities in the PWN that are reasonable only for young systems in the so called free-expansion phase (the first few thousands years). It moreover imposes constraints on the kind of injected particle's distribution (a power-law). This model has been further extended to take partially into account the interaction with the SNR (Reynolds and Chanan 1984; Reynolds and Chevalier 1984), or changes in injection history (Atoyan 1999). More recently numerical models have been developed (Bednarek and Bartosik 2003; Bucciantini et al. 2011; Gelfand et al. 2009; Martín et al. 2012; Tanaka and Takahara 2010, 2013; Torres et al. 2013). Using a numerical approach, the evolution can be followed for a longer time taking into account the various phases of the interaction with the parent SNR. These models allow us, not only to include a complex evolutionary history, but also to model the evolution of distribution functions, that are not necessarily a single power law (either broken power laws have been considered, or a power-law plus a Maxwellian).
In particular, the work by Gelfand et al. (2009) is exemplary for showing the richness of behaviors that is found even within the 1-Zone treatment, and the degree of complexity that one can investigate using simple tools.

The main advantage of 1-Zone models is their easiness of implementation, and the fact that they allow us to sample a large parameter space of often unknown injection or evolutionary properties. Of course one should not expect more than a rough quantitative agreement with the global observed properties of these system. In particular these models are not reliable for the high-energy X-ray emission properties, because, as it will be shown later, they are strongly affected by the internal flow dynamics, that these kinds of models fail completely to consider. On the other hand the Radio emission properties, the location of spectral breaks, the ratio of synchrotron to Inverse Compton emission, can be fairly well modelled. The same holds for the overall evolution, where the typical duration and timescale of the various phases of interaction with the SNR are correctly recovered.

Given that quite often the quality of the available data is limited, 1-Zone models turn out to be perhaps the only justifiable approach. On the other hand they also constitute a good starting point for more accurate, multidimensional studies.

\section{1-D Models}

It is a general expectation that, increasing the dimensionality of a model, should lead to a more realistic description of a system: a description closer to the true working. If one includes also a simple spatial dependence of the various quantities (often a dependence on the radial coordinate from the pulsar) it is possible to develop $1-D$ models (or equivalently $1+1$ models). The first 1-D model of the Crab Nebula was put forward by Rees and Gunn (1974). Already this simple description allows one to understand some of the spatial features/properties of this object. The under-luminous region, centered on the location of the pulsar, is interpreted as the ultra-relativistic unshocked wind. The wavelength dependent size of the Crab Nebula, with radio emission extending further outside the X-ray one, is explained in term of combined bulk flow from the termination shock to the outer edge of the nebula, and synchrotron cooling. The existence of a bright torus is understood as due to compression of the magnetic field in the outer layers of the nebula. This model also showed that it was in principle possible to use the nebular properties to infer the conditions of the pulsar wind, like its Lorentz factor and/or magnetization. This model has been further developed by Kennel and Coroniti (1984a,b) who solved the full set of relativistic MHD equations, and provided a quantitative estimate of the structural properties of the Crab Nebula. Later Emmering and Chevalier (1987) presented a time dependent analytical solution.

Further development of 1-D models came mainly from numerical investigation, which allowed us to avoid many of 
the simplifications required by analytical treatment. Blondin et al. (2001) and van der Swaluw et al. (2001) were the first to investigate the dynamics of the PWN-SNR interaction using a hydrodynamical code. All the various phases of the mutual interaction where followed: the free-expansion phase (Chevalier and Fransson 1992), the reverberation phase, and the final Sedov phase. Similar works have also been carried out by Bucciantini et al. (2004b, 2003) which were the first to use relativistic MHD and to investigate the role of magnetic field. More recently de Jager et al. (2008) have used a 1-D model to compute the evolution of an injected distribution function, and to compute an integrated spectrum for G21.5-0.9. Latest results have been presented by Vorster et al. (2013).

One of the major issues raised by 1-D models is the so called $\sigma$ problem (Melatos 1998). Traditionally the $\sigma$ problem mixes together two different problems: one related to the efficiency of accelerating particles at a shock, the other to the ability of slowing down a relativistic magnetized outflow. The latter is a dynamical problem, and in the following I will only consider this aspect. In Ideal MHD a radial highly-magnetized (with a toroidal field) supersonic relativistic pulsar wind cannot be slowed down by a shock to match the typical expansion speeds of the confining SNR. For this to happen it is required a small value of the magnetization (Arons 2004). In fact, if the ratio of Poynting flux to total energy flux in the pulsar wind exceeds $\sim 10^{-3}$, a quasi-stable shock cannot form, and one expects a subsonic solution for the pulsar outflow, a breeze. This contrasts strongly the expectations of pulsar wind electrodynamics (Beskin et al. 1998; Bogovalov 2001; Goldreich and Julian 1970; Michel 1969), which suggest that the magnetization in the wind, at best, with minor deviations from radial outflow, could be $\sim 1$ at the typical distance of the nebulae (Chiueh et al. 1998). A closer issue is the inability to reproduce the relative size of X-ray an Radio in the Crab Nebula, and similar young systems (Amato et al. 2000), and the correct softening of the spectrum with the distance from the pulsar (Reynolds 2004). All of these problems are related to the fact that in 1-D models there is a one-to-one correspondence between distance, age, magnetic field and flow velocity. This inevitably leads to onion-like structures, which are the exact opposite of the complete mixing at the base of 1-Zone models.

\section{2-D Models}

It is evident that, in the presence of a toroidal magnetic field, stresses will arise in the nebula, leading to a prolate shape. Begelman and Li (1992) were the first to develop a 2-D model accounting for this effect, in an attempt to explain the observed prolate geometry of the Crab Nebula, and to use it as a further constrain on the plasma magnetization. Models that assume a cylindrical geometry are referred as $2-D$ models (or equivalently $2+1$ models).
However the interest in multidimensional models for PWNe received a considerable boost only at the start of the 21st century. Optical and X-ray images from HST, CHANDRA and XMM-Newton have shown that the inner region of PWNe is characterized by a complex axisymmetric structure, generally referred as jet-torus structure. First observed in the Crab (Hester et al. 1995; Weisskopf et al. 2000), it has been detected in many other PWNe (Camilo et al. 2004; Gaensler et al. 2002; Lu et al. 2002; Pavlov et al. 2003; Romani and Ng 2003; Romani et al. 2005; Slane et al. 2004), and there is a general consensus that such features are always present in young systems. However given the complex dynamics, associated with the larger degrees of freedom due to the increased dimensionality, only the use of numerical codes for computational fluid dynamics (Del Zanna et al. 2003; Komissarov 1999) allowed us to move beyond the simple analytical solution by Begelman and $\mathrm{Li}$ (1992). van der Swaluw (2003) was the first to present a numerical model of a prolate nebula applied to 3C58. It was however the investigation of the dynamics of the pulsar wind that drove most of the successive attempts at modeling the inner jet-torus structure. Theoretical (Begelman and Li 1994; Beskin et al. 1998) and numerical (Bogovalov 2001; Bucciantini et al. 2006; Contopoulos et al. 1999; Komissarov 2006; Spitkovsky 2006; Timokhin 2006) studies of relativistic winds from pulsars agree that, at the typical distance of the termination shock, the wind luminosity and magnetization are not uniform, but can be described by the so called split monopole solution: most of the energy is confined to the equatorial region, and there is no evidence of jets or collimated pulsar outflows along the axis. It is reasonable to expect that such a wind could drive a complex dynamics at least in the inner region. Bogovalov and Khangoulian (2002a); Bogovalov and Khangoulyan (2002b) were the first to investigate this problem, modeling the flow dynamics at the termination shock. Later Lyubarsky (2002) suggested that hoop stresses in the body of the nebula could lead to the collimation of the jet. This has been extensively confirmed numerically by different groups (Bogovalov et al. 2005; Del Zanna et al. 2004, 2006; Komissarov and Lyubarsky 2004; Volpi et al. 2008). This numerical work has proved quite rich in its results, and various aspects of the nebular morphology have been investigated, from the details of emission maps (Del Zanna et al. 2006), to polarization (Bucciantini et al. 2005b), to the overall spectrum (Volpi et al. 2008). We are now in a position where 2-D models can accurately reproduce the observed X-ray features of the Crab Nebula, including the wisps and the knot, in terms of relative size and brightness. More recently attention has focused of the problem of time variability. Variability in the X-ray was known since the first observations. In particular the variability in the wisps region (Hester et al. 1995; Schweizer et al. 2013). 2-D models have shown that such variability can be reproduced (Bucciantini 2008; Bucciantini and Del Zanna 2006; Camus et al. 2009) both in term of its typical timescale and in its morphological pattern, of outgoing waves. Interest- 
ingly these 2-D models have shown that the limiting value of the wind magnetization $\sigma$, required to explain the observed morphology and the presence of a jet, is about one order of magnitude higher than the maximum value allowed in 1-D models, and this mostly because the extra degree of freedom of the 2-D geometry allows to accommodate more magnetized winds. There is also a large degree of internal fluid turbulence and mixing. This strongly contrasts the basic assumption of 1-D models (the onion-like structure), and is suggestive that reality could be closer to the full mixing assumption of 1-Zone models.

Apart from the dynamics of the internal region, 2-D models have been also applied to the interaction with the SNR. Jun (1998) and Bucciantini et al. (2004a) were the first to investigate the Rayleigh-Taylor instability at the interface with the SNR ejecta, both in the magnetized an unmagnetized case, showing the development of the filamentary network observed in the Crab Nebula. Blondin et al. (2001) were the first to investigate the 2-D dynamics of the reverberation phase, showing the high level of mixing between the SNR and PWN, and the role of density gradients in the ISM, leading to a relic PWN displaced with respect to the pulsar, as it is often observed in old systems. van der Swaluw et al. (2004) investigated in detail the interaction of a pulsar moving across the SNR, the transition from spherical PWNe in the early phase to a cometary structure, and in van der Swaluw et al. (2002) the interaction with the SNR forward shock was also taken into account. Ferreira and de Jager (2008) analyzed the role of ISM density gradients, in the presence of an ordered magnetic field.

\section{3-D Models}

It has been known for a long time (Begelman 1998; Nalewajko and Begelman 2012) that configurations with a purely toroidal magnetic field are subject to current driven instability (kink instability). Indeed a kink-like instability is seen in PWNe (Mori et al. 2004; Pavlov et al. 2003). To fully capture the dynamics associated with this instability it is necessary to develop full 3-D models (also known as $3+1$ models). Recently this process has been studied via numerical simulations in the simplified regime of a magnetized column confined by a hot atmosphere (Mizuno et al. 2011, 2012; O'Neill et al. 2012). These simulations have shown that the instability reaches the non-linear regime on a typical Alfvènic timescale, and that, after about 10 Alfvènic timescales, the original toroidal field has almost completely vanished. While this suggests a very efficient way out of the $\sigma$ problem (Begelman 1998), on the other hand it is obvious that such a violent instability cannot operate in PWNe, because polarization measures (Bietenholz 1991; Smith et al. 1988; Velusamy 1985) constrain the level of ordered magnetic field to be very high. Part of the reason for this inconsistency can be found in the simplified regime that these models consider. In PWNe the toroidal magnetic field is continuously injected by the pulsar wind, and the PWN is not pressure confined but confined by a high density wall at the contact discontinuity with the SNR ejecta. On one hand this implies that there must be a balance between the injection and dissipation of the toroidal field, on the other hand, it implies that in the inner region, close to the termination shock, where the plasma is injected, the magnetic field is bound to be close to toroidal, while instabilities would only act in the outer regions. Unfortunately a full 3-D simulation of a PWN, evolving long enough to reach a balance, has not been carried out yet, mostly because of computational cost.

However recently Porth et al. (2013) presented a very interesting preliminary study, where the dynamics of the PWN in a full 3-D setting was investigated for a time-length corresponding to $70 \mathrm{yr}$. While this is about one order of magnitude smaller than the age of the Crab Nebula, and it can be debated if the nebula has reached a state of dynamical equilibrium, nevertheless this timescale is longer that a typical Alfvènic crossing time, and longer than the synchrotron cooling time for X-ray emitting particles. The results confirm the qualitative expectations: the inner region close to the termination shock is still dominated by a toroidal field, and preserves the axisymmetric structure of 2D simulations; a jet is formed and extends in the body of the nebula; instabilities at the outer edge of the nebula act to reduce the net toroidal field component. It is also found that the nebula can accommodate even a wind with high $\sigma$, of order unity. Recalling that, even minor deviations of the wind from a purely radial flow, can lower $\sigma$ to values of order unity, at the typical distance of the termination shock radius (Chiueh et al. 1998), this suggests that the $\sigma$ problem is likely an artifact of the 1-D geometry of theories of old. However this does not rule out the possibility of other non-ideal effects, like dissipation and/or reconnection to play a role.

\section{Bow-Shocks}

As we pointed out in the previous sections, few works have been devoted to the multi-dimensional investigation of the dynamical evolution of old PWNe inside the SNR. Interestingly there are several works focusing on the interaction with the ISM. Pulsars moving in the ISM produce the so called pulsar wind bow-shock nebulae (Bell et al. 1995; Cordes et al. 1993; Gaensler et al. 2004; Kargaltsev et al. 2008; Ng et al. 2012; Pavlov et al. 2006). The first attempt to model the physics of these systems, accounting for the presence of neutral Hydrogen in the ISM, was done by Bucciantini (2002b); Bucciantini and Bandiera (2001) extending the thin-layer approximation (Giuliani 1982) used to model cometary nebulae (Bandiera 1993; Wilkin 1996, 2000). The thin layer approximation is conceptually analogous to a 1-D model, given that it neglects the thickness of the nebula, and all quantities depend only on the distance from the apex. These models however provide a quite good description of the head region of these nebulae in terms of shape, thickness to hydrogen penetration, $\mathrm{H}_{\alpha}$ luminosity, as was later confirmed by more accurate 2-D axisymmet- 
ric simulations both in the hydrodynamical regime (Bucciantini 2002a; Gaensler et al. 2004) and in the relativistic MHDl regime (Bucciantini et al. 2005a). A 3-D extension of the study of these systems to take into account either a nonuniform ambient medium, or the anisotropy in the pulsar wind energy flux (in analogy with 2-D simulations of Crablike PWNe) has been presented by Vigelius et al. (2007).

More recently bow-shock models have also been developed for the interaction and confinement of the pulsar wind in binary systems (Bogovalov et al. 2012, 2008; BoschRamon et al. 2012). In this case it is the wind from the companion that provides the confining medium. These models have been used to explain orbital variations and modulations of the high energy comptonized emission.

Acknowledgements. The author wishes to thank his many collaborators, among whom: Luca Del Zanna, Elena Amato, Jonathan Arons, Rino Bandiera, Serguei Komissarov, that have all substantially contributed to the development of this field.

\section{References}

Amato, E. and Arons, J.: 2006, ApJ 653, 325.

Amato, E., Salvati, M., Bandiera, R., Pacini, F., and Woltjer, L.: 2000, A\&A 359, 1107.

Arons, J.: 2004, in F. Camilo and B.M. Gaensler, eds., Young Neutron Stars and Their Environments, vol. 218 of IAU Symposium, 163.

Arons, J. and Tavani, M.: 1994, ApJS 90, 797.

Asaoka, I. and Koyama, K.: 1990, PASJ 42, 625.

Atoyan, A.M.: 1999, A\&A 346, L49.

Atoyan, A.M. and Aharonian, F.A.: 1996, MNRAS 278, 525.

Bandiera, R.: 1993, A\&A 276, 648.

Bandiera, R.: 2000, in P.C.H. Martens, S. Tsuruta, and M.A. Weber, eds., Highly Energetic Physical Processes and Mechanisms for Emission from Astrophysical Plasmas, vol. 195 of IAU Symposium, 189.

Barnes, A. and Scargle, J.D.: 1973, ApJ 184, 251.

Bednarek, W. and Bartosik, M.: 2003, A\&A 405, 689.

Begelman, M.C.: 1998, ApJ 493, 291.

Begelman, M.C. and Li, Z.Y.: 1992, ApJ 397, 187.

Begelman, M.C. and Li, Z.Y.: 1994, ApJ 426, 269.

Bell, J.F., Bailes, M., Manchester, R.N., Weisberg, J.M., and Lyne, A.G.: 1995, ApJ 440, L81.

Beskin, V.S., Kuznetsova, I.V., and Rafikov, R.R.: 1998, MNRAS 299, 341.

Bietenholz, M.F.: 1991, A Radio Study of the Crab Nebula., Ph.D. thesis, UNIVERSITY OF TORONTO (CANADA).

Blondin, J.M., Chevalier, R.A., and Frierson, D.M.: 2001, ApJ 563, 806.

Bogovalov, S.V.: 1997, A\&A 327, 662.

Bogovalov, S.V.: 2001, A\&A 371, 1155.

Bogovalov, S.V., Chechetkin, V.M., Koldoba, A.V., and Ustyugova, G.V.: 2005, MNRAS 358, 705.

Bogovalov, S.V. and Khangoulian, D.V.: 2002a, MNRAS 336, L53.

Bogovalov, S.V. and Khangoulyan, D.V.: 2002b, Astronomy Letters $28,373$.

Bogovalov, S.V., Khangulyan, D., Koldoba, A.V., Ustyugova, G.V., and Aharonian, F.A.: 2012, MNRAS 419, 3426.
Bogovalov, S.V., Khangulyan, D.V., Koldoba, A.V., Ustyugova, G.V., and Aharonian, F.A.: 2008, MNRAS 387, 63.

Bosch-Ramon, V., Barkov, M.V., Khangulyan, D., and Perucho, M.: 2012, A\&A 544, A59.

Bucciantini, N.: 2002a, A\&A 387, 1066.

Bucciantini, N.: 2002b, A\&A 393, 629.

Bucciantini, N.: 2008, in C. Bassa, Z. Wang, A. Cumming, and V.M. Kaspi, eds., 40 Years of Pulsars: Millisecond Pulsars, Magnetars and More, vol. 983 of American Institute of Physics Conference Series, 186-194.

Bucciantini, N., Amato, E., Bandiera, R., Blondin, J.M., and Del Zanna, L.: 2004a, A\&A 423, 253.

Bucciantini, N., Amato, E., and Del Zanna, L.: 2005a, A\&A 434, 189.

Bucciantini, N., Arons, J., and Amato, E.: 2011, MNRAS 410, 381.

Bucciantini, N. and Bandiera, R.: 2001, A\&A 375, 1032.

Bucciantini, N., Bandiera, R., Blondin, J.M., Amato, E., and Del Zanna, L.: 2004b, A\&A 422, 609.

Bucciantini, N., Blondin, J.M., Del Zanna, L., and Amato, E.: 2003, A\&A 405, 617.

Bucciantini, N. and Del Zanna, L.: 2006, A\&A 454, 393.

Bucciantini, N., del Zanna, L., Amato, E., and Volpi, D.: 2005b, A\&A 443, 519.

Bucciantini, N., Metzger, B.D., Thompson, T.A., and Quataert, E.: 2012, MNRAS 419, 1537.

Bucciantini, N., Quataert, E., Arons, J., Metzger, B.D., and Thompson, T.A.: 2008, MNRAS 383, L25.

Bucciantini, N., Quataert, E., Metzger, B.D., Thompson, T.A., Arons, J., and Del Zanna, L.: 2009, MNRAS 396, 2038.

Bucciantini, N., Thompson, T.A., Arons, J., Quataert, E., and Del Zanna, L.: 2006, MNRAS 368, 1717.

Bykov, A.M. and Treumann, R.A.: 2011, Astronomy and Astrophysics Review19, 42.

Camilo, F., Gaensler, B.M., Gotthelf, E.V., Halpern, J.P., and Manchester, R.N.: 2004, ApJ 616, 1118.

Camus, N.F., Komissarov, S.S., Bucciantini, N., and Hughes, P.A.: 2009, MNRAS 400, 1241.

Chevalier, R.A.: 2004, Advances in Space Research 33, 456.

Chevalier, R.A. and Fransson, C.: 1992, ApJ 395, 540.

Chiueh, T., Li, Z.-Y., and Begelman, M. C., 1998, ApJ , 505, 835

Contopoulos, I., Kazanas, D., and Fendt, C.: 1999, ApJ 511, 351.

Cordes, J.M., Romani, R.W., and Lundgren, S.C.: 1993, Nature 362, 133.

Dall'Osso, S., Stratta, G., Guetta, D., Covino, S., De Cesare, G., and Stella, L.: 2011, A\&A 526, A121.

de Jager, O.C., Ferreira, S.E.S., and Djannati-Ataï, A.: 2008, in F.A. Aharonian, W. Hofmann, and F. Rieger, eds., American Institute of Physics Conference Series, vol. 1085 of American Institute of Physics Conference Series, 199-202.

Del Zanna, L., Amato, E., and Bucciantini, N.: 2004, A\&A 421, 1063.

Del Zanna, L., Bucciantini, N., and Londrillo, P.: 2003, A\&A 400, 397.

Del Zanna, L., Volpi, D., Amato, E., and Bucciantini, N.: 2006, A\&A 453, 621.

Emmering, R.T. and Chevalier, R.A.: 1987, ApJ 321, 334.

Ferreira, S.E.S. and de Jager, O.C.: 2008, A\&A 478, 17.

Gaensler, B.M., Arons, J., Kaspi, V.M., Pivovaroff, M.J., Kawai, N., and Tamura, K.: 2002, ApJ 569, 878.

Gaensler, B.M. and Slane, P.O.: 2006, ARA\&A 44, 17. 
Gaensler, B.M., van der Swaluw, E., Camilo, F., Kaspi, V.M., Baganoff, F.K., Yusef-Zadeh, F., and Manchester, R.N.: 2004, ApJ 616, 383.

Gelfand, J.D., Slane, P.O., and Zhang, W.: 2009, ApJ 703, 2051.

Giuliani, Jr., J.L.: 1982, ApJ 256, 624.

Goldreich, P. and Julian, W.H.: 1969, ApJ 157, 869.

Goldreich, P. and Julian, W.H.: 1970, ApJ 160, 971.

Harding, A.K.: 1996, Spac.Scienc.Review75, 257.

Hester, J.J.: 2008, ARA\&A 46, 127.

Hester, J.J., Scowen, P.A., Sankrit, R., et al.: 1995, ApJ 448, 240.

Hibschman, J.A. and Arons, J.: 2001, ApJ 560, 871.

Jun, B.I.: 1998, ApJ 499, 282.

Kargaltsev, O., Misanovic, Z., Pavlov, G.G., Wong, J.A., and Garmire, G.P.: 2008, ApJ 684, 542.

Kargaltsev, O. and Pavlov, G.G.: 2010, X-ray Astronomy 2009; Present Status, Multi-Wavelength Approach and Future Perspectives 1248, 25.

Kennel, C.F. and Coroniti, F.V.: 1984a, ApJ 283, 694.

Kennel, C.F. and Coroniti, F.V.: 1984b, ApJ 283, 710.

Komissarov, S.S.: 1999, MNRAS 303, 343.

Komissarov, S.S.: 2006, MNRAS 367, 19.

Komissarov, S.S. and Lyubarsky, Y.E.: 2004, MNRAS 349, 779.

Lu, F.J., Wang, Q.D., Aschenbach, B., Durouchoux, P., and Song, L.M.: 2002, ApJ 568, L49.

Lyubarsky, Y. and Liverts, M.: 2008, ApJ 682, 1436.

Lyubarsky, Y.E.: 2002, MNRAS 329, L34.

Lyubarsky, Y.E.: 2003, MNRAS 345, 153.

Martín, J., Torres, D.F., and Rea, N.: 2012, MNRAS 427, 415.

Melatos, A. 1998, MemSAIt, 69, 1009

Michel, F.C.: 1969, ApJ 158, 727.

Mizuno, Y., Lyubarsky, Y., Nishikawa, K.I., and Hardee, P.E.: 2011, ApJ 728, 90.

Mizuno, Y., Lyubarsky, Y., Nishikawa, K.I., and Hardee, P.E.: 2012, ApJ 757, 16.

Mori, K., Burrows, D.N., Pavlov, G.G., Hester, J.J., Shibata, S., and Tsunemi, H.: 2004, in F. Camilo and B.M. Gaensler, eds., Young Neutron Stars and Their Environments, vol. 218 of IAU Symposium, 181.

Nalewajko, K. and Begelman, M.C.: 2012, MNRAS 427, 2480.

Ng, C.Y., Bucciantini, N., Gaensler, B.M., Camilo, F., Chatterjee, S., and Bouchard, A.: 2012, ApJ 746, 105.

O'Neill, S.M., Beckwith, K., and Begelman, M.C.: 2012, MNRAS 422, 1436.

Pacini, F. and Salvati, M.: 1973, ApJ 186, 249.

Pavlov, G.G., Sanwal, D., and Zavlin, V.E.: 2006, ApJ 643, 1146.

Pavlov, G.G., Teter, M.A., Kargaltsev, O., and Sanwal, D.: 2003, ApJ 591, 1157.

Porth, O., Komissarov, S.S., and Keppens, R.: 2013, MNRAS 431, L48.

Rees, M.J. and Gunn, J.E.: 1974, MNRAS 167, 1.

Reynolds, S.: 2004, in J.P. Paillé, ed., 35th COSPAR Scientific Assembly, vol. 35 of COSPAR Meeting, 3572.

Reynolds, S.P. and Chanan, G.A.: 1984, ApJ 281, 673.

Reynolds, S.P. and Chevalier, R.A.: 1984, ApJ 278, 630.

Romani, R.W. and Ng, C.Y.: 2003, ApJ 585, L41.

Romani, R.W., Ng, C.Y., Dodson, R., and Brisken, W.: 2005, ApJ 631, 480.

Schweizer, T., Bucciantini, N., Idec, W., Nilsson, K., Tennant, A., Weisskopf, M., and Zanin, R.: 2013, ArXiv e-prints .

Sironi, L. and Spitkovsky, A.: 2009, ApJ 698, 1523.

Sironi, L. and Spitkovsky, A.: 2011a, ApJ 741, 39.
Sironi, L. and Spitkovsky, A.: 2011b, ApJ 726, 75.

Slane, P., Helfand, D.J., van der Swaluw, E., and Murray, S.S.: 2004, ApJ 616, 403.

Smith, F.G., Jones, D.H.P., Dick, J.S.B., and Pike, C.D.: 1988, MNRAS 233, 305.

Spitkovsky, A.: 2006, ApJ 648, L51.

Tanaka, S.J. and Takahara, F.: 2010, ApJ 715, 1248.

Tanaka, S.J. and Takahara, F.: 2013, MNRAS 429, 2945.

Timokhin, A. N.: 2006, MNRAS , 368, 1055

Torres, D.F., Cillis, A.N., and Martín Rodriguez, J.: 2013, ApJ 763, L4.

van der Swaluw, E.: 2003, A\&A 404, 939.

van der Swaluw, E., Achterberg, A., and Gallant, Y.A.: 2002, in P.O. Slane and B.M. Gaensler, eds., Neutron Stars in Supernova Remnants, vol. 271 of Astronomical Society of the Pacific Conference Series, 135.

van der Swaluw, E., Achterberg, A., Gallant, Y.A., and Tóth, G.: 2001, A\&A 380, 309.

van der Swaluw, E., Downes, T.P., and Keegan, R.: 2004, A\&A 420, 937.

Velusamy, T.: 1985, MNRAS 212, 359.

Vigelius, M., Melatos, A., Chatterjee, S., Gaensler, B.M., and Ghavamian, P.: 2007, MNRAS 374, 793.

Volpi, D., Del Zanna, L., Amato, E., and Bucciantini, N.: 2008, A\&A 485, 337.

Vorster, M.J., Ferreira, S.E.S., de Jager, O.C., and Djannati-Ataï, A.: 2013, A\&A 551, A127.

Weiler, K.W. and Panagia, N.: 1978, A\&A 70, 419.

Weisskopf, M.C., Hester, J.J., Tennant, A.F., et al.: 2000, ApJ 536, L81.

Wilkin, F.P.: 1996, ApJ 459, L31.

Wilkin, F.P.: 2000, ApJ 532, 400. 